\title{
Mechanically robust cylindrical metal terahertz waveguides for cryogenic applications
}

Robert Wallis

Riccardo Degl'Innocenti

Oleg Mitrofanov

Joanna Waldie

Carlos M. Bledt

Jeffrey E. Melzer

James A. Harrington

Harvey E. Beere

David A. Ritchie 


\title{
Mechanically robust cylindrical metal terahertz waveguides for cryogenic applications
}

\author{
Robert Wallis*a , Riccardo Degl'Innocenti ${ }^{\mathrm{a}}$, Oleg Mitrofanov ${ }^{\mathrm{b}}$, Joanna Waldie ${ }^{\mathrm{a}}$, Carlos M. Bledt ${ }^{\mathrm{c}}$, \\ Jeffrey E. Melzer ${ }^{\mathrm{c}}$, James A. Harrington ${ }^{\mathrm{c}}$, Harvey E. Beere ${ }^{\mathrm{a}}$, David A. Ritchie ${ }^{\mathrm{a}}$ \\ ${ }^{a}$ Cavendish Laboratory, University of Cambridge, J J Thomson Avenue, Cambridge, CB3 OHE, UK; \\ ${ }^{b}$ Department of Electronic and Electrical Engineering, University College London, Torrington Place, \\ London, WC1E 7JE, UK; ' School of Engineering, Rutgers University, 607 Taylor Road, Piscataway, \\ New Jersey 08854, USA.
}

\begin{abstract}
As the ambition behind $\mathrm{THz}$ quantum cascade laser based applications continues to grow, abandoning free-space optics in favor of waveguided systems promises major improvements in targeted, easy to align, and robust radiation delivery. This is especially true in cryogenic environments, where illumination is traditionally challenging. Although the field of $\mathrm{THz}$ waveguides is rapidly developing, most designs have limitations in terms of mechanical stability at low temperatures, and are costly and complicated to fabricate to lengths $>1 \mathrm{~m}$. In this work, we investigate readily available cylindrical metal waveguides which are suitable for effective power delivery in cryogenic environments, and explore the optimal dimensions and materials available. The materials chosen were extruded un-annealed and annealed copper, as well as stainless steel, with bore diameters of $1.75,2.5$, and $4.6 \mathrm{~mm}$. Measurements were performed at three different frequencies, 2.0, 2.85 and $3.2 \mathrm{THz}$, with optimal transmission losses $<3 \mathrm{~dB} / \mathrm{m}$ demonstrated at $2.0 \mathrm{THz}$. Additionally, novel optical couplers are also presented and characterised, with the ability to change the beam path by $90^{\circ}$ with a coupling loss of just $2.2 \mathrm{~dB}$ whilst maintaining mode quality, or thermally isolate sections of waveguide with a coupling loss as low as $0.5 \mathrm{~dB}$. The work presented here builds on previous work ${ }^{1}$, and forms a comprehensive investigation of cryogenically compatible $\mathrm{THz}$ waveguides and optical couplers, paving the way for a new generation of systems to utilize THz QCLs for a host of low-temperature investigations.
\end{abstract}

Keywords: Terahertz waveguides, quantum cascade laser, mode profile, beam shaping, cryogenics, optical components

\section{INTRODUCTION}

The field of terahertz $(\mathrm{THz})$ waveguides has recently seen significant progress, both in improving key performance characteristics, such as fabrication techniques, transmission losses, and dispersion ${ }^{2}$, whilst also integrating components into $\mathrm{THz}$ applications that previously utilized free space optics ${ }^{3,4}$. Multiple benefits follow from this, for instance the ability to achieve purgeable, targeted power delivery, which can be easily aligned over long distances $(>100 \mathrm{~mm})^{5}$. There currently exists a considerable range of waveguide designs, including hollow ${ }^{6}$ and solid ${ }^{7}$ core dielectric designs, photonic crystal $^{8}$, wire ${ }^{9}$, coplanar ${ }^{10}$, hollow core metallic ${ }^{11,12}$, and dielectric lined metallic ${ }^{13,14}$. Relatively little attention however has been placed on $\mathrm{THz}$ waveguides capable of operating in cryogenic environments $(<4 \mathrm{~K})$. In this area, there exists the potential for the study of numerous quantum systems ${ }^{15,16}$ operating with transitions on the THz scale $(1.0-5.0 \mathrm{THz} \approx 4-$ $20 \mathrm{meV}$ ). However, the energy of $1 \mathrm{THz}$ radiation corresponds to $\sim 50 \mathrm{~K}$, necessitating the use of liquid helium cooling in order to avoid thermal smearing of the transitions of interest.

In this work, attention is directed towards developing waveguides and optical hardware suitable for such environments. Delivery of $\mathrm{THz}$ radiation in cryogenic environments has been challenging to date, as operation below $4 \mathrm{~K}$ is usually hindered by multiple cryogenic vacuum chambers, which each require the use of an absorbing optical window. To circumvent this challenge, a waveguide based delivery system presents itself as a promising solution. In these environments, the transmission loss becomes crucial, to compensate for the often significant physical distance between source and sample. For illuminating the sample, a Gaussian-like beam is desirable, and in addition, the temperature

*rw497@cam.ac.uk; phone +44 1223 765579;

Terahertz, RF, Millimeter, and Submillimeter-Wave Technology and Applications X, edited by

Laurence P. Sadwick, Tianxin Yang, Proc. of SPIE Vol. 10103, 1010307 · @ 2017 SPIE

CCC code: $0277-786 \mathrm{X} / 17 / \$ 18 \cdot$ doi: $10.1117 / 12.2250530$ 
variations involved in thermal cycling makes mechanical stability another central consideration. From a thermal point of view, it is also crucial to limit the additional heat load introduced into the sample space by the introduction of the waveguide. As a result, the thermal conductivity of the material chosen is key. This issue can also be addressed by the inclusion of suitable connecting structures if necessary, which are specifically designed for thermal isolation. One design which appears to encompass these factors is the cylindrical hollow metallic waveguide (HMWG), the study of which has previously been limited to diameters $<1 \mathrm{~mm}^{11,17}$, rendering the losses too high for the distances involved in cryogenic applications. Designs for flexible HMWGs also exist, by coating a dielectric tube with an inner metallic layer ${ }^{18}$. However, the dielectric materials used makes them potentially mechanically unstable at cryogenic temperatures. In this work, the focus is on demonstrating the applicability of extruded hollow metal waveguides for cryogenic $\mathrm{THz}$ applications. This is followed by the demonstration of two types of optical couplers designed to efficiently manipulate the beam direction, as well as reduce the thermal conduction of the optical system, thus enabling the construction of waveguided $\mathrm{THz}$ delivery systems in cryogenic environments with limited heat load on the sample space.

\section{HOLLOW METAL WAVEGUIDES}

\subsection{Experimental setup}

The HMWGs chosen for investigation were made of un-annealed copper (UnCu) and annealed copper ( $\mathrm{AnCu}$ ), and stainless steel (SS). Copper was chosen due to its high electrical conductivity, and resulting low skin depth, which gives a minimal optical penetration and thus ohmic losses. Other strengths include being both readily malleable, and available in a variety of dimensions to suit the application. Stainless steel was also included, as its low thermal conductivity renders it useful in many component designs at cryogenic temperatures. All the waveguides were extruded cylinders, with three different inner diameters, $1.75,2.5$, and $4.6 \mathrm{~mm}$, and wall thicknesses of $0.71,1.13$, and $0.87 \mathrm{~mm}$, respectively. These were chosen to balance the trade-off between single mode transmission, at the expense of increasing losses with reducing diameter ${ }^{18}$. A set of three HMWGs of a particular material and diameter were prepared into lengths between 50 and $150 \mathrm{~mm}$, and cleaned with subsequent submersions in acetone, IPA, $\mathrm{HCl}$ and $\mathrm{HNO}_{3}$ solutions to remove any surface oxides, residual annealing scale, and tarnish ${ }^{19}$.
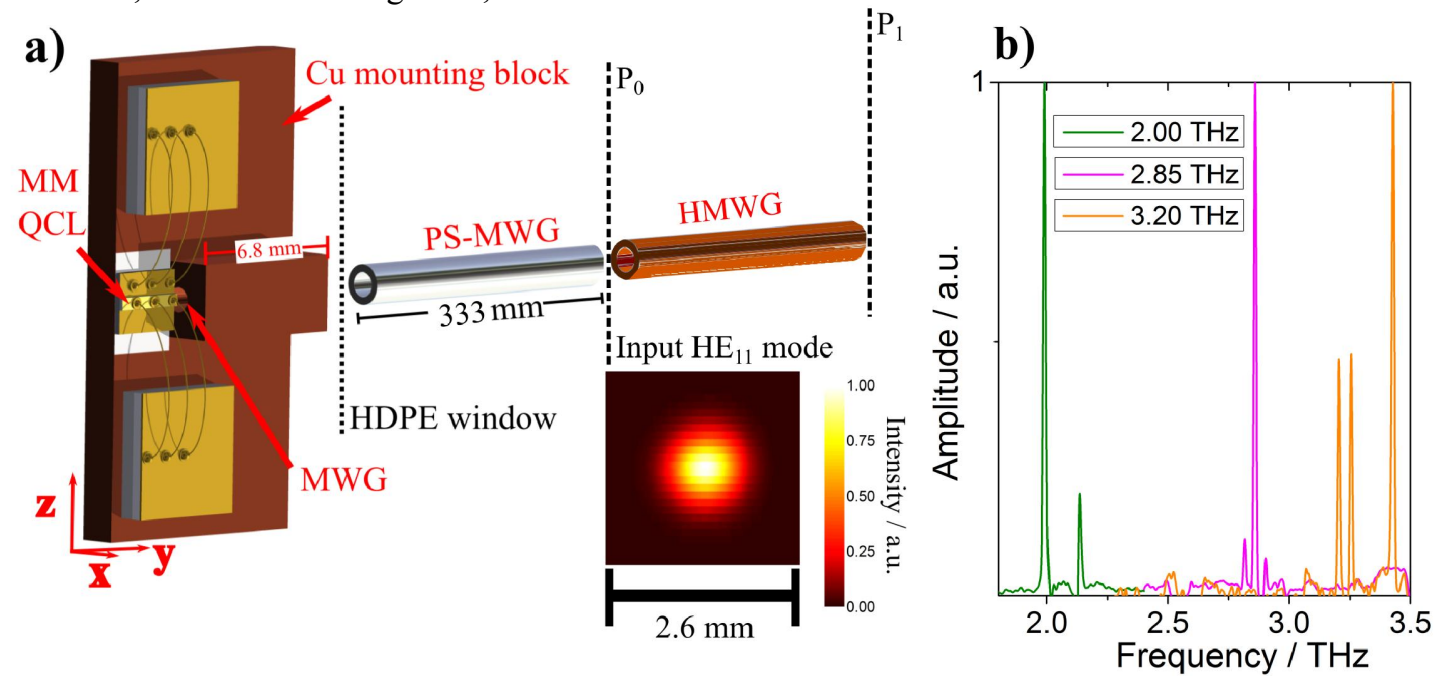

Figure 1. a) Schematic of the setup used to characterize the HMWGs. A QCL was placed within the cryostat, and coupled into the external PS-MWG through a cylindrical HDPE window of $1.35 \mathrm{~mm}$ wall thickness. Initial and final powers were measured from the end of the PS-MWG $\left(\mathrm{P}_{0}\right)$ and from the end of the HMWG $\left(\mathrm{P}_{1}\right)$ respectively using a Golay cell detector. Inset - Representative beam profile from the PS-MWG when excited using the $2.0 \mathrm{THz}$ QCL, showing the Gaussian-like $\mathrm{HE}_{11}$ mode used as a launch beam. b) Frequency spectra for the three QCL devices used to characterize the HMWGs, showing quasi-single mode operation at $2.0 \mathrm{THz}$ (green), and $2.85 \mathrm{THz}$ (pink), and multimode emission between 3.2 and 3.4 $\mathrm{THz}$ (orange).

The THz quantum cascade laser $(\mathrm{QCL})^{20}$ was chosen as a radiation source due to its high output power ${ }^{21}$, compactness, and frequency selectivity ${ }^{22}$. To bring the radiation from the QCL to the waveguide under test, whilst cleaning and normalizing the beam, a $333 \mathrm{~mm}$ long, $1 \mathrm{~mm}$ diameter flexible polystyrene-lined hollow metallic waveguide (PSMWG) ${ }^{23}$ was used, as has been previously reported ${ }^{5,24}$. The full set up is shown in Fig. 1 a). The QCLs were pulsed 
between 10 and $100 \mathrm{kHz}$, with $2 \%$ duty cycle. Radiation was coupled into the external PS-MWG, followed by the HMWG under test. Initial and final intensities were recorded from the end of the PS-MWG $\left(\mathrm{P}_{0}\right)$ and HMWG $\left(\mathrm{P}_{1}\right)$ respectively, via the use of a Golay cell detector and lock-in detection at $24 \mathrm{~Hz}$. QCLs emitting at 2.0. 2.85 and $\sim 3.2-$ 3.4 THz were used, in order to investigate the HMWG performance over a range of frequencies (see Fig. $1 \mathrm{~b}$ )). Before coupling into a test waveguide, the beam profile from the PS-MWG was examined for each device. A representative profile is shown in the inset of Fig. 1 a) for the $2.0 \mathrm{THz}$ QCL, exhibiting the fundamental Gaussian-like HE $\mathrm{H}_{11}$ mode of the structure. This presented an excellent initial beam with which to couple effectively into the HMWGs. Three lengths of each waveguide were measured for a given frequency, bore diameter, and material type, with lengths of 50, 100, and $150 \mathrm{~mm}$ forming a set, to distinguish the coupling losses from the transmission losses.

\subsection{Results}

The initial and final powers were recorded for each of the three lengths of waveguide in a given set, allowing the loss to be determined as a function of length. A linear fit then provided both the coupling loss from the intercept, and the transmission loss from the gradient. An exemplary graph of the transmission loss as a function of length measured for the $4.6 \mathrm{~mm}$ diameter UnCu waveguide is shown in Fig. 2, using the $2.0 \mathrm{THz}$ QCL. To control for atmospheric absorption, measurements were taken with the waveguides purged with nitrogen gas. The transmission losses in $\mathrm{dB} / \mathrm{m}$ for all the waveguide testing are shown in Table 1 . These varied considerably, from up to $\sim 30 \mathrm{~dB} / \mathrm{m}$ for a $1.75 \mathrm{~mm} \mathrm{UnCu}$ waveguide, down to $<3 \mathrm{~dB} / \mathrm{m}$ for the $4.6 \mathrm{~mm} \mathrm{AnCu}$ waveguide set. This value is comparable to previously reported, but more structurally complex designs ${ }^{20}$, and is sufficiently low to allow such a waveguide to be effectively implemented into waveguide based cryogenic delivery systems with path lengths in excess of 1 meter, whilst retaining enough signal for measurements. From Table 1, there are three main trends to be observed.

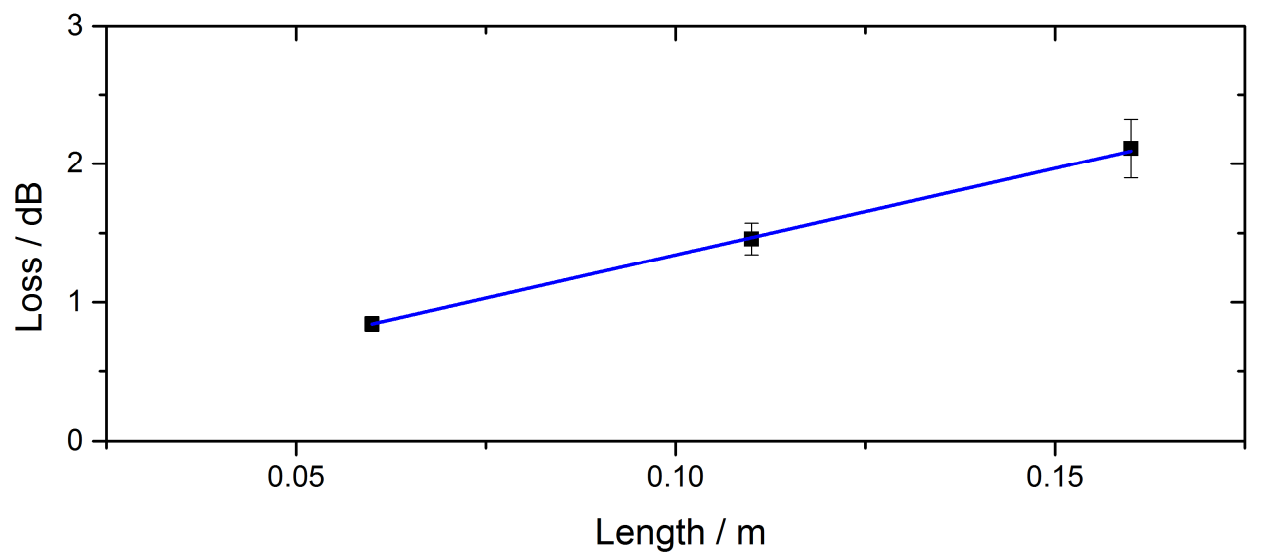

Figure 2. Exemplary plot of the waveguide loss as a function of length for the $4.6 \mathrm{~mm}$ diameter UnCu waveguide, measured at $2.0 \mathrm{THz}$. A linear fit provides the coupling loss from the intercept, and the transmission losses from the gradient. All measurements were taken whilst nitrogen purging.

Table 1. Summary of transmission losses in $\mathrm{dB} / \mathrm{m}$ for various materials, diameters, and frequencies. All measurements were taken whilst nitrogen purging.

\begin{tabular}{|c|c|c|c|c|c|c|c|c|c|}
\hline & \multicolumn{3}{|c|}{$1.75 \mathrm{~mm}$} & \multicolumn{3}{|c|}{$2.5 \mathrm{~mm}$} & \multicolumn{3}{|c|}{$4.6 \mathrm{~mm}$} \\
\hline & $2.0 \mathrm{THz}$ & $2.85 \mathrm{THz}$ & $3.2 \mathrm{THz}$ & $2.0 \mathrm{THz}$ & $2.85 \mathrm{THz}$ & $3.2 \mathrm{THz}$ & $2.0 \mathrm{THz}$ & $2.85 \mathrm{THz}$ & $3.2 \mathrm{THz}$ \\
\hline UnCu & $15.7 \pm 1.1$ & $23.0 \pm 2.5$ & $30.2 \pm 2.7$ & $9.2 \pm 1.7$ & $10.0 \pm 2.0$ & $17.0 \pm 1.7$ & $10.2 \pm 1.6$ & $10.0 \pm 4.0$ & $20.4 \pm 1.8$ \\
\hline $\mathrm{AnCu}$ & $1.6 \pm 2.0$ & $7.0 \pm 1.2$ & $20.9 \pm 1.9$ & & & & $0.5 \pm 1.6$ & $2.0 \pm 1.3$ & $3.7 \pm 1.0$ \\
\hline SS & $10.2 \pm 1.8$ & $15 \pm 2.2$ & $23.0 \pm 2.6$ & & & & & & \\
\hline
\end{tabular}

For an increase in bore diameter, the transmission loss reduces, for a fixed frequency and material. This is typically ascribed to a larger fraction of the mode propagating inside the hollow core of the waveguide for larger bore diameters $^{18,20}$. The second is a consistent increase in losses for a given bore diameter and material, as the frequency is 
increased. The third is that the $\mathrm{AnCu}$ waveguides appears to possess a reliably lower loss than the equivalent unannealed version. For example, for a $1.75 \mathrm{~mm}$ diameter at $2.0 \mathrm{THz}$, the loss is reduced by $\sim 10 \mathrm{~dB} / \mathrm{m}$ by moving to the $\mathrm{AnCu}$ waveguide. This knowledge is extremely useful when considering the construction of any future cryogenic delivery system.

\section{WAVEGUIDE COUPLERS}

Having investigated $\mathrm{THz}$ waveguides that are mechanically suitable for cryogenic applications, the focus now turns to developing waveguide couplers to facilitate the construction of a full cryogenic delivery system. Optical couplers are desirable for a number of reasons. Often, separate sections of waveguide are required to be incorporated together in specific orientations, depending on the geometry of the particular setup considered. To do this, couplers are required to connect the separate sections, doing so in an efficient and simple manner. Additionally, it is necessary to retain the thermal isolation of various sections of the cryostat assembly, as far as possible, which necessitates isolating breaks in the metal waveguide delivery system, whilst also minimizing optical losses. Such components would allow a continuation of the drive towards fully waveguided, 'plug and play' optical setups in the THz, doing away with the need for free space components, which are often time consuming to align, possess a considerable footprint on the optical table, and have the potential to introduce considerable losses if not correctly aligned.

In the work presented here, we choose to focus on two efficient coupling components, which are highly likely to be required for any practical cryogenic application. These are a $90^{\circ}$ coupler, which allows waveguides orientated perpendicular to each other to be efficiently coupled together with minimal loss, as well as a thermal isolating straight coupler, allowing the splicing together of multiple HMWGs efficiently, whilst simultaneously minimizing thermal conduction between the individual sections. This latter consideration is of primary importance when cryogenic applications are considered, due to the high thermal conductivity of the copper used to construct the waveguides. This has the potential to introduce non-trivial thermal loads into the cryogenic sample chamber, with the associated reduction in the operating performance of the cryostat in question, if not addressed effectively.

\subsection{Modelling and design}

The design and theoretical modelling of the $90^{\circ}$ coupler will be considered first. An optimal design needs to encompass a number of considerations. It should possess dimensions which are suitable for the environment in which it will be used, and constructed from materials which will not be detrimentally affected mechanically by any potentially low-temperature environments it is placed in. An effective component should also have the ability to be 'plug and play', such that there is a minimum time cost associated with setting up and aligning the waveguides under consideration, and additionally, would allow some degree of interchangeability between different waveguide types and outer diameters. Over multiple use cycles, any critical parts would also ideally be replaceable, without the requirement to replace the entire component.

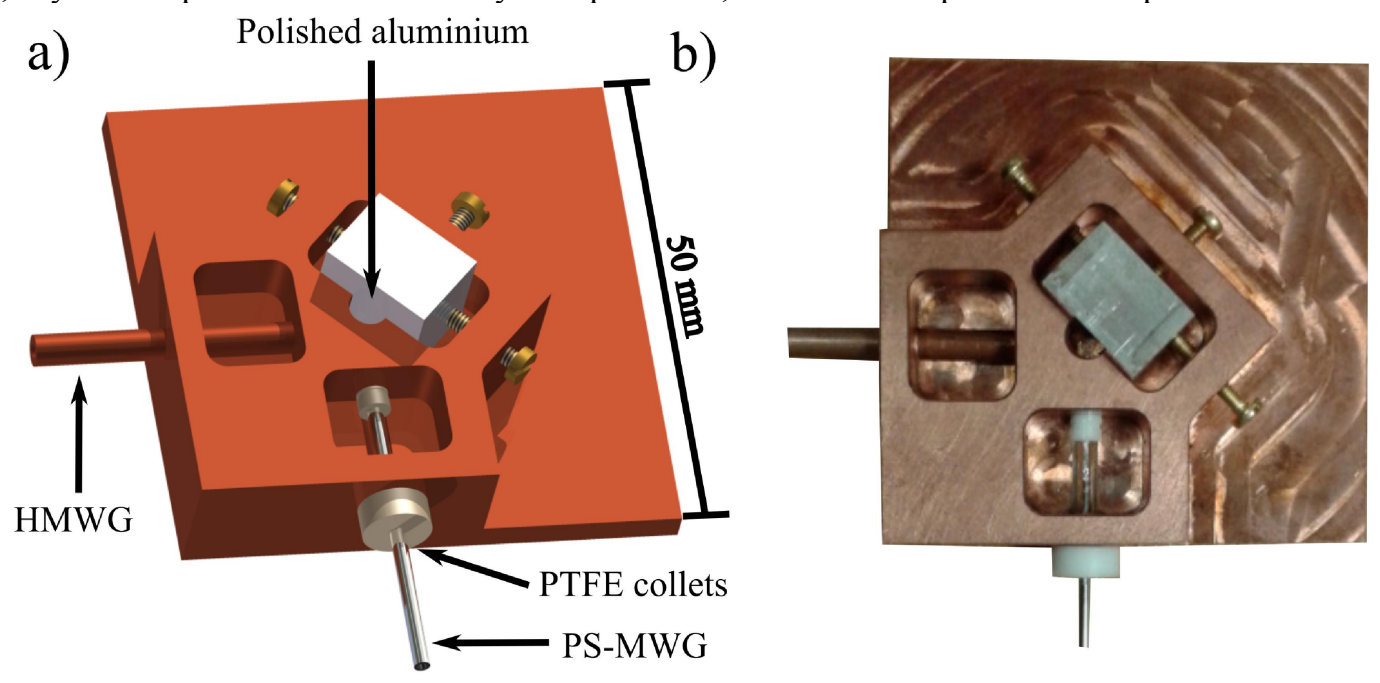

Figure 3. a) Schematic of the $90^{\circ}$ coupling component, shown here with a $1.7 \mathrm{~mm}$ outer diameter PS-MWG delivering the incoming $\mathrm{THz}$ beam, and a $3.17 \mathrm{~mm}$ outer diameter $\mathrm{Cu}$ HMWG collecting the beam, after reflection from the polished aluminium block. b) Optical photograph of the coupler. 
The overall device is shown in Fig. 3 a), with a total footprint of $50 \mathrm{~mm}^{2}$. The body was fabricated from copper, with $3.17 \mathrm{~mm}$ outer diameter cylindrical perpendicular cavities to accommodate the two waveguides to be coupled. As a reflecting element to guide the light between waveguides, a polished aluminium block was placed at $45^{\circ}$ to the incoming beam axis. The final effective $6 \mathrm{~mm}$ of each waveguide is made from the copper of the component body, the final 100 $\mu \mathrm{m}$ of the outer diameter of which was reamed, to give as smooth an inner surface as possible. Additionally, in order to allow the component to be used in conjunction with both a $3.17 \mathrm{~mm}$ outer diameter $\mathrm{Cu}$ HMWG, and a $1.7 \mathrm{~mm}$ outer diameter PS-MWG, a pair of PTFE collets were fabricated, to allow matching of the outer diameters of the waveguides with the fabricated bore of the component, as necessary. An optical photograph of the coupler is shown in Fig. 3 b).

The component performance from an optical perspective was also modelled using finite element analysis software, in order to check the expected nature of the transfer of optical power between waveguides (see Fig. 4). A 10 x $10 \mathrm{~mm}$ area was defined, encompassing the entry and exit waveguides, which were defined as air under ambient conditions, as well as the reflecting aluminium surface, positioned at $45^{\circ}$ to the incoming beam. Everything else was defined as the copper of the component body. The width of the waveguides was defined as $2.3 \mathrm{~mm}$. In order to simulate the $\mathrm{THz}$ radiation, a pulse was defined with a Gaussian envelope in both the travelling and lateral directions, acting as a good approximation to the modes supported in these types of waveguide (see Fig. 1 a)), with full width half maximum of 2 ps, and $2 \mathrm{~mm}$ respectively. Fig. 4 shows six separate frames, captured at 5, 20, 25, 30, 35, and 44 ps, showing how the pulse propagates through the system. At $5 \mathrm{ps}$, the pulse enters the initial waveguide from the left of the frame, and starts to be reflected by the polished aluminium surface by $25 \mathrm{ps}$. At $30 \mathrm{ps}$, the pulse starts to be collected by the exit waveguide at $90^{\circ}$, and by $44 \mathrm{ps}$, it can be observed that the pulse has been effectively transferred to the exit waveguide, with the lateral Gaussianlike power distribution preserved. This suggests that the fundamental $\mathrm{TE}_{11}$ mode of the HMWGs can be preserved using such a component to modify the beam by $90^{\circ}$.
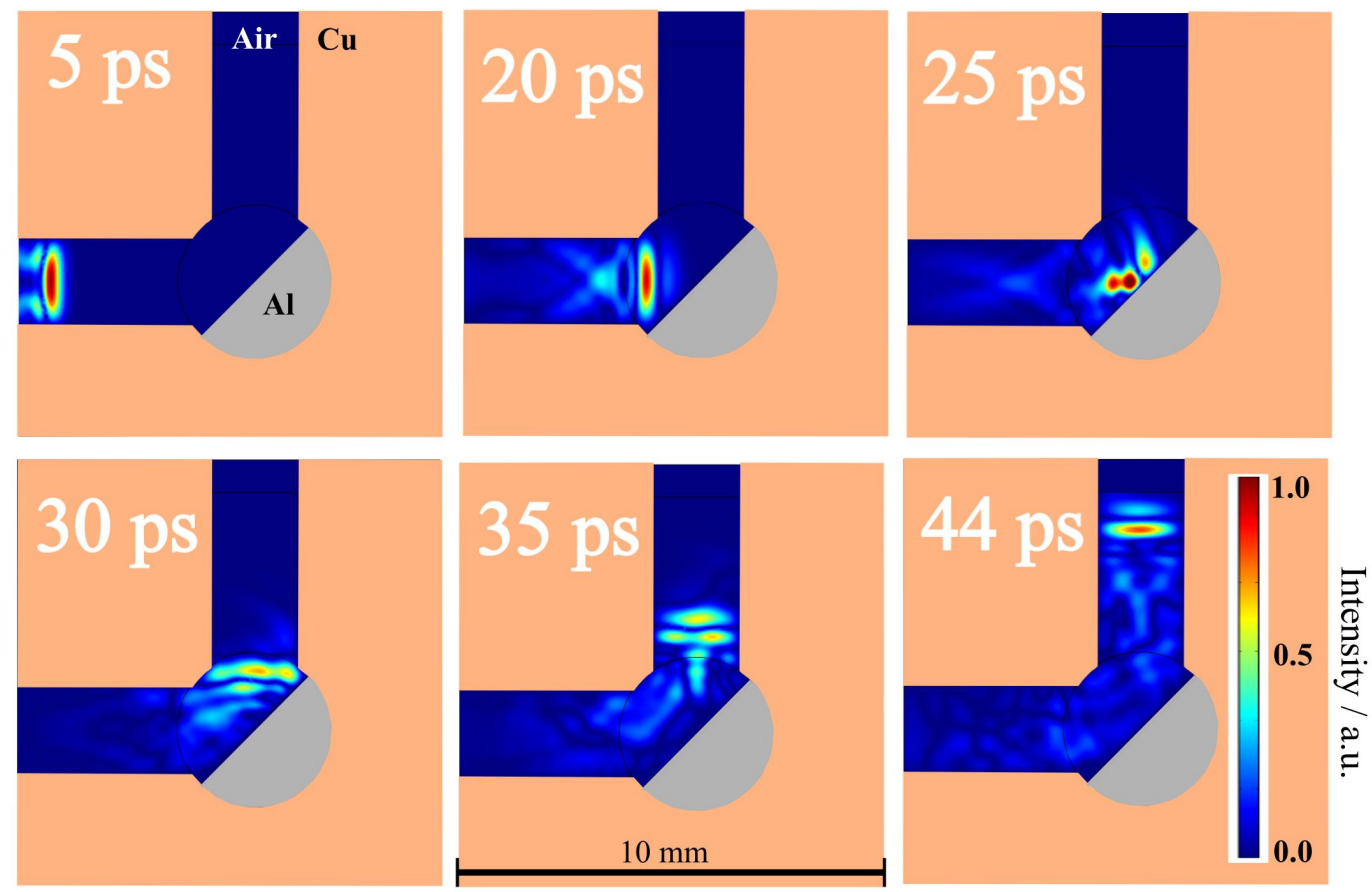

Figure 4. Finite element analysis simulations used to model the $90^{\circ}$ coupler. A $10 \times 10 \mathrm{~mm}$ area was defined to represent the area of the coupler where the reflection of the beam occurs. The diameter of the input and exit waveguides is $2.3 \mathrm{~mm}$, and the aluminium reflector is placed at an angle of $45^{\circ}$. The THz pulse can be seen entering the structure at 5 ps, with reflection off the aluminium interface occurring at $25 \mathrm{ps}$. By $44 \mathrm{ps,} \mathrm{the} \mathrm{pulse} \mathrm{has} \mathrm{successfully} \mathrm{entered} \mathrm{the} \mathrm{exit} \mathrm{waveguide,} \mathrm{with} \mathrm{the}$ lateral power distribution maintained.

The second coupler considered was a thermally isolating straight coupler, allowing two sections of HMWG to be secured together, maintaining the efficient transfer of the $\mathrm{THz}$ beam whilst simultaneously minimizing the thermal contact between the two sections. This was achieved using a single part component, as shown in Fig. 5. The two $6.35 \mathrm{~mm}$ outer 
diameter $\mathrm{Cu}$ HMWGs insert into either end of the coupler, which has a total length of $32 \mathrm{~mm}$. A spacer layer, fabricated with the same $4.6 \mathrm{~mm}$ inner diameter as the HMWGs is incorporated at the point where the waveguides would meet, with a thickness of $2 \mathrm{~mm}$, in order to avoid contact between the highly conductive waveguides, and provide thermal isolation to any heat flux flowing down the waveguides induced by potential thermal gradients within the cryogenic system.

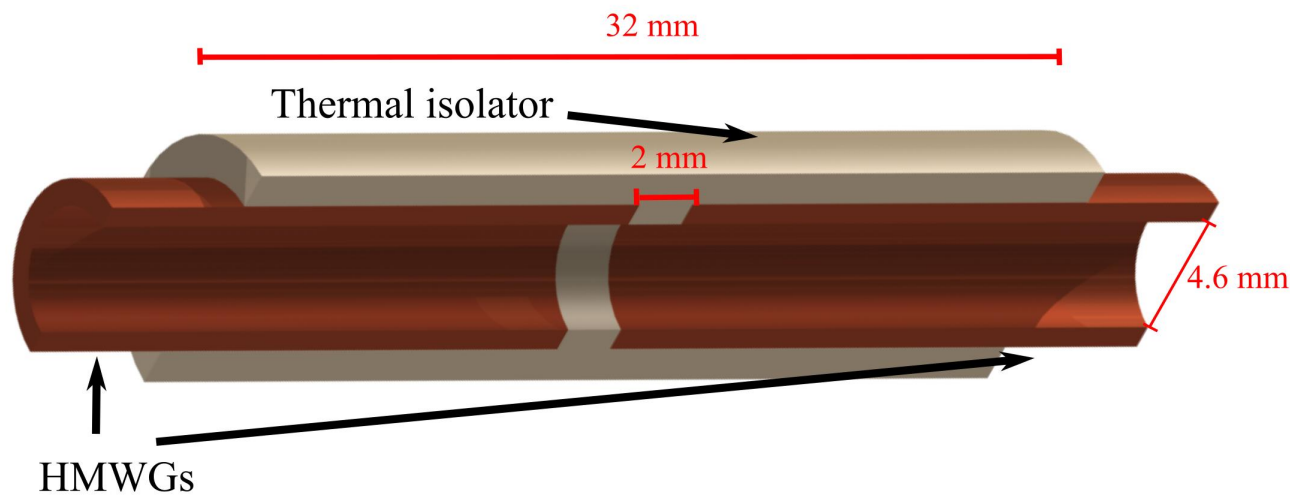

Figure 5. Cut-through schematic of the straight thermal isolating coupler design. Two $6.35 \mathrm{~mm}$ outer diameter HMWGs insert into the coupler, and are separated by a $2 \mathrm{~mm}$ thick spacer layer, with matching inner diameter, which acts to increase the thermal isolation of the waveguides.

The material chosen for the thermal isolating coupler was $\mathrm{HYSOL}^{\circledR}$, a commercially available material designed for cryogenic applications where thermal isolation is required. The material has a number of favourable properties. These include a low thermal conductivity $(<3 \mathrm{~W} / \mathrm{mK})$, high mechanical stability over a full range of cryogenic temperatures $(<$ $1 \mathrm{~K}$ to $>300 \mathrm{~K}$ ), and over successive thermal cycles. It is also easily machinable, allowing ease of fabrication of the requisite parts, as well as mechanically rigid, enabling the two waveguides sections to remain optically aligned at all temperatures.

\subsection{Results}

Once fabricated, these two coupler designs were characterized at room temperature in order to assess their performance in comparison to the desired optical and mechanical specifications outlined above. In order to test the THz properties of the $90^{\circ}$ coupler, a setup similar to that shown in Fig. 1 was adopted. A QCL emitting at $2.85 \mathrm{THz}$ was coupled into a 333 $\mathrm{mm}$ long PS-MWG in order to clean the beam and provide an input waveguide for characterization. First, the coupling efficiency of the $90^{\circ}$ coupler was established. This was achieved by measuring the initial power, exiting the PS-MWG, using a Golay cell and lock-in detection at $24 \mathrm{~Hz}$. The $90^{\circ}$ coupler was then introduced onto the end of the PS-MWG, and a $100 \mathrm{~mm}$ length of $1.75 \mathrm{~mm}$ inner diameter AnCu HMWG with known loss was introduced into the exit aperture of the coupler. The final power was then recorded in a similar manner. Accounting for the losses in the HMWG, a coupling efficiency of $50 \pm 5 \%$ was measured, suggesting that a considerable degree of the optical power can be transferred between two perpendicular waveguides using such an optical coupler. It was estimated that the primary loss mechanisms contributing to this value included the free carrier losses from the copper body, as well as scattering losses from the inner waveguide surfaces, Additionally, some imperfections in the polished aluminium reflecting face may play a role, as well as some degree of radiation loss due to the divergence of the temporarily unconstrained beam in the reflection area. This value was compared to an alternative method of deflecting the beam by $90^{\circ}$ - that of bending a HMWG. This was achieved by bending a $15 \mathrm{~mm}$ length of $1.75 \mathrm{~mm}$ inner diameter $\mathrm{AnCu}$ with a bend radius of $15.9 \mathrm{~mm}$, using a standard tube bender to deform the waveguide in a ductile fashion. The loss introduced by such a bend was measured in a similar manner, yielding a transmission of $60 \pm 5 \%$. Such a value suggests that the amount of power transferred is comparable to using the $90^{\circ}$ coupler. However, such a simple bend technique possesses a number of drawbacks in comparison. These include the requirement to individually bend each piece of waveguide as required, introducing a degree of non-reversible human error into the system assembly for each bend, as well as losing the ability to use two separate types and diameters of waveguide to create the desired beam deflection. Another advantage of the coupler is the 'plug and play' nature of the design, such that both ends of the two waveguides used are automatically aligned and mechanically secured within any larger setup.

Another key consideration for such a coupler is the beam profile resulting from the $90^{\circ}$ change in direction. In order to characterize this, as well as compare to a standard $90^{\circ}$ bend in the waveguide, far fields were acquired at a distance of 
$4.5 \mathrm{~mm}$, using a Golay cell with $1 \mathrm{~mm}$ aperture, scanned in the plane perpendicular to the beam. The results are shown in Fig. 6 .

a)

b)
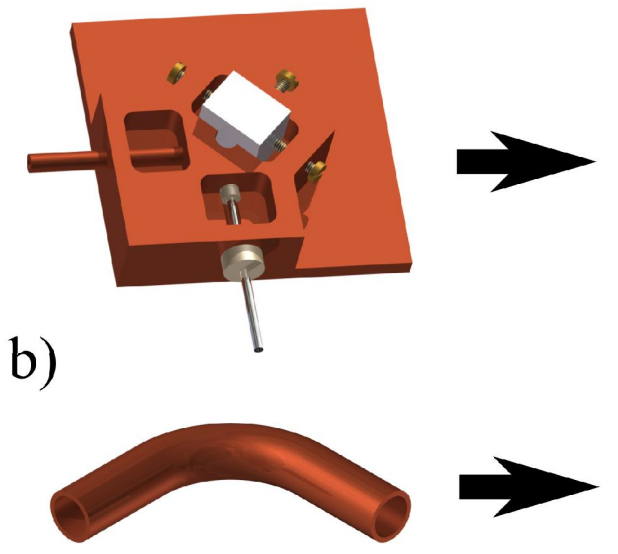

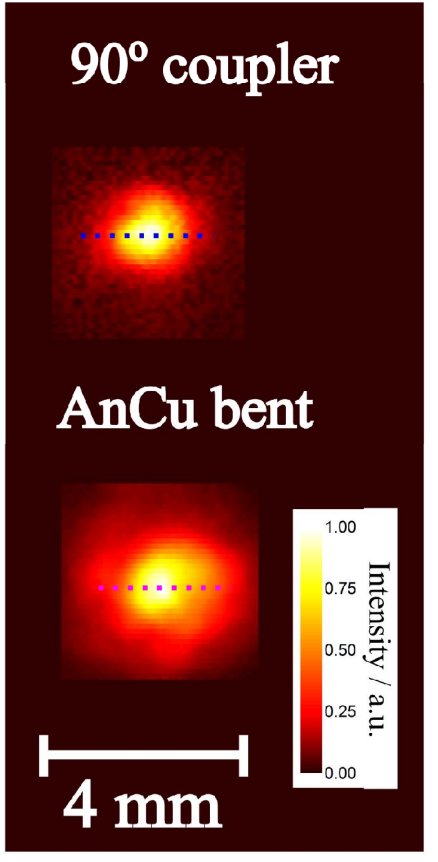

AnCu bent c)

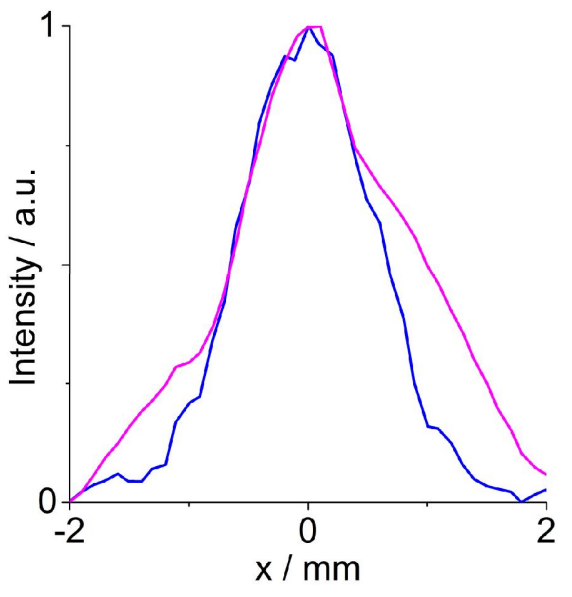

Figure 6. a) Beam profile acquired using the $90^{\circ}$ coupler, showing a Gaussian-like distribution of the optical power b) Beam profile acquired using a $1.75 \mathrm{~mm}$ inner diameter $\mathrm{AnCu} \mathrm{HMWG}$ bent to $90^{\circ}$ with a bend radius of $15.9 \mathrm{~mm}$. Both were acquired using a Golay cell with $1 \mathrm{~mm}$ aperture, at a distance of $4.5 \mathrm{~mm}$. c) Line-cuts of the $90^{\circ}$ coupler (blue dashed line) and bent $\mathrm{AnCu}$ waveguide (pink dashed line) profiles, showing a lower divergence beam for the $90^{\circ}$ coupler.

Fig. 6 a) displays the beam profile acquired using the $90^{\circ}$ coupler, showing a Gaussian-like distribution of the optical power. Fig. 6 b) shows the beam profile acquired using a $1.75 \mathrm{~mm}$ inner diameter AnCu HMWG bent to $90^{\circ}$, with a bend radius of $15.9 \mathrm{~mm}$. As can be seen, the beam profile is again Gaussian-like, however with a slightly increased spatial extent in both horizontal and vertical directions (Fig. 6 c)). From a cryogenic systems standpoint, the two beam profiles are effectively equivalent, both being of sufficient quality to be incorporated efficiently into any larger system as required.

Additionally, the coupling efficiency of the straight thermal isolating waveguide coupler was measured. A QCL emitting at $2.85 \mathrm{THz}$ was coupled into a $333 \mathrm{~mm}$ long PS-MWG in order to clean the beam. This was then directly coupled into a $50 \mathrm{~mm}$ long, $4.6 \mathrm{~mm}$ diameter AnCu HMWG, whose entrance was placed in the same plane as the exit aperture of the PS-MWG. The power emitted from the end of this HMWG was then recorded using a Golay cell and lock-in detection at $24 \mathrm{~Hz}$, which formed the initial power for the coupling measurement. The thermal isolating spacer was then placed onto the end of the first HMWG, and another $50 \mathrm{~mm}$ long, $4.6 \mathrm{~mm}$ diameter AnCu HMWG inserted into the exit end, with the final power being recorded. Accounting for the losses of the two HMWGs, a coupling efficiency $>90 \%$ was recorded, suggesting that such a coupler can be implemented in future systems with minimal loss introduced to the THz beam path.

\section{CONCLUSION}

In this work, the performance of potential $\mathrm{THz}$ cryogenic system components have been investigated. These include cylindrical hollow copper waveguides with inner diameters between 1.75 and $4.6 \mathrm{~mm}$, as well as optical couplers to both redirect the beam path by $90^{\circ}$, and to thermally isolate and connect successive waveguide sections. For the waveguides, transmission losses $<3 \mathrm{~dB} / \mathrm{m}$ at $2.0 \mathrm{THz}$ have been observed for a $\mathrm{N}_{2}$ purged $4.6 \mathrm{~mm}$ bore diameter annealed $\mathrm{Cu}$ waveguide, and for the couplers, it has been shown that the beam direction can be modified by $90^{\circ}$, with a coupling 
efficiency of $50 \%$, whilst still maintaining mode quality, and waveguides can be spliced together with efficiencies $>$ $90 \%$, whilst simultaneously introducing a thermal isolation effect. The results presented here pave the way for a new generation of cryogenic applications requiring efficient and targeted power delivery in the $\mathrm{THz}$ regime.

\section{REFERENCES}

[1] Wallis, R., Degl'Innocenti, R., Jessop, D. S., Mitrofanov, O., Bledt, C. M., Melzer, J. E., Harrington, J. A., Beere, H. E. and Ritchie, D. A., "Investigation of hollow cylindrical metal terahertz waveguides suitable for cryogenic environments," Opt. Express 24(26), 30002-30014 (2016).

[2] Mitrofanov, O., James, R., Fernandez F. A., Mavrogordatos T. K. and Harrington J. A., "Reducing transmission losses in hollow THz waveguides," Terahertz Science and Technology, IEEE Transactions on 1(1), 124-132 (2011).

[3] Awad M. M. and Cheville, R. A., "Transmission terahertz waveguide-based imaging below the diffraction limit," Appl. Phys. Lett. 86(22), 221107 (2005).

[4] Zhan, H., Mendis, R. and Mittleman, D. M., "Superfocusing terahertz waves below $\lambda / 250$ using plasmonic parallel-plate waveguides," Opt. Express 18(9), 9643-9650 (2010).

[5] Wallis, R., Degl'Innocenti, R., Jessop, D. S., Ren, Y., Klimont, A., Shah, Y. D., Mitrofanov, O., Bledt, C. M., Melzer, J. E., Harrington, J. A., Beere, H. E. and Ritchie, D. A., "Efficient coupling of double-metal terahertz quantum cascade lasers to flexible dielectric-lined hollow metallic waveguides," Opt. Express 23(20), 2627626287 (2015).

[6] Hidaka, T., Minamide, H., Ito, H., Maeta, S-I. and Akiyama, T., "Ferroelectric PVDF Cladding Terahertz Waveguide," Proc. of SPIE 5135, 70-77 (2003).

[7] Nagel, M., Marchewka, A. and Kurz, H., "Low-index discontinuity terahertz waveguides," Opt. Express 14(21), 9944-9954 (2006).

[8] Goto, M., Quema, A., Takahashi, M., Ono, S. and Sarukura, N., "Teflon Photonic Crystal Fiber as Terahertz Waveguide," Jap. Journal of Appl. Phys. 43(28), 317-319 (2004).

[9] Wang K. and Mittleman, D. M., "Metal wires for terahertz wave guiding," Nature 432(7015), 376-379 (2004).

[10] Grischkowsky, D. R., "Optoelectronic characterization of transmission lines and waveguides by terahertz timedomain spectroscopy," IEEE Journal of Selected Topics in Quant. Elect. 6(6), 1122-1135 (2000).

[11] McGowan, R. W., Gallot, G. and Grischkowsky, D., "Propagation of ultrawideband short pulses of terahertz radiation through submillimeter-diameter circular waveguides," Opt. Letters 24(20), 1431-1433 (1999).

[12] Doradla, P. and Giles, R. H., "Dual-frequency characterization of bending losses in hollow flexible terahertz waveguides," Proc. SPIE 8985, Terahertz, RF, Millimeter, and Submillimeter-Wave Technology and Applications VII, 898518 (2014).

[13] Mitrofanov, O. and Harrington, J. A., "Dielectric-lined cylindrical metallic THz waveguides: mode structure and dispersion," Opt. Express 18(3), 1898-1903 (2010).

[14] Doradla, P., Joseph, C. S., Kumar, J. and Giles, R. H., "Characterization of bending loss in hollow flexible terahertz waveguides," Opt. Express 20(17), 19176-19184 (2012).

[15] Shibata, K., Umeno, A., Cha, K. M. and Hirakawa, K., "Photon-Assisted Tunneling through Self-Assembled InAs Quantum Dots in the Terahertz Frequency Range," PRL 109(7), 077401 (2012).

[16] Yoshida, K., Shibata, K. and Hirakawa, K., "Terahertz Field Enhancement and Photon-Assisted Tunneling in Single-Molecule Transistors," PRL 115(13), 138302 (2015).

[17] Gallot, G., Jamison, S. P., McGowan, R. W. and Grischkowsky, D., "Terahertz waveguides," Journal of the Optical Society of America B 17(5), 851-863 (2000).

[18] Harrington, J. A., George, R., Pedersen, P. and Mueller, E., "Hollow polycarbonate waveguides with inner $\mathrm{Cu}$ coatings for delivery of terahertz radiation," Opt. Express 12(21), 5263-5268 (2004).

[19] Davis, J. R., [Copper and Copper Alloys], ASM International, (2001)

[20] Köhler, R., Tredicucci, A., Beltram, F., Beere, H. E., Linfield, E. H., Davies, A. G., Ritchie, D. A., Iotti R. C. and Rossi, F., "Terahertz semiconductor-heterostructure laser," Nature 417(6885), 156-159 (2002).

[21]Li, L., Chen, L., Zhu, J., Freeman, J., Dean, P., Valavanis, A., Davies, A. G. and Linfield, E. H., "Terahertz quantum cascade lasers with $>1$ W output powers," Elec. Letters 50(4), 309-311 (2014). 
[22] Freeman, J. R., Brewer, A., Madéo, J., Cavalié, P., Dhillon, S. S., Tignon, J., Beere H. E. and Ritchie, D. A., "Broad gain in a bound-to-continuum quantum cascade laser with heterogeneous active region," Appl. Phys. Lett. 99(24), 241108 (2011).

[23] Navarro-Cia, M., Vitiello, M. S., Bledt, C. M., Melzer, J. E., Harrington J. A. and Mitrofanov, O., "Terahertz wave transmission in flexible polystyrene-lined hollow metallic waveguides for the 2.5-5 THz band," Opt. Express 21(20), 23748-23755 (2013).

[24] Degl'Innocenti, R., Shah, Y. D., Jessop, D. S., Ren, Y., Mitrofanov, O., Beere, H. E. and Ritchie, D. A., "Hollow metallic waveguides integrated with terahertz quantum cascade lasers," Opt. Express 22(20), 2443924449 (2014). 